\title{
Some Recent Advances in Complex Networks Synchronization ${ }^{\star}$
}

\author{
Guanrong Chen, Xiaofan Wang, Xiang Li, and Jinhu Lü
}

\begin{abstract}
The current study of complex dynamical networks is pervading almost all kinds of science, engineering and technology, ranging from mathematics to computers, physics to biology, even to sociology. Its impacts on the modern high-tech industries, financial markets and human life are prominent and will be far-reaching. Research on fundamental properties and dynamical features of such complex networks has indeed become overwhelming.

This Chapter presents a brief overview of some past and current studies on the subject of complex dynamical network synchronization, particularly from an engineering and technological perspective. Some commonly concerned issues in the current research of network synchronization, mainly on
\end{abstract}

Guanrong Chen

Department of Electronic Engineering, City University of Hong Kong,

Hong Kong SAR, China

e-mail: gchen@ee.cityu.edu.hk

Xiaofan Wang

Department of Automation, Shanghai Jiao Tong University, Shanghai 200240, China

e-mail: $x f w a n g @ s j t u . e d u . c n$

Xiang Li

Department of Electronic Engineering, Fudan University, Shanghai 200433, China e-mail: lix@fudan.edu.cn

Jinhu Lü

Institute of Systems Science, Academy of Mathematics and Systems Science, Chinese Academy of Sciences, Beijing 100190, China

e-mail: jhlu@iss.ac.cn

* This work was supported by the NSFC-HKRGC Joint Research Scheme under Grant N-CityU 107/07, the Hong Kong Research Grants Council under the GRF Grant CityU 1117/08E, the National Natural Science Foundation of China under Grants 60821091 and 60772158, the National Basic Research (973) Program of China under Grant 2007CB310805, the Important Direction Project of Knowledge Innovation Program of Chinese Academy of Sciences under Grant KJCX3SYW-S01, and the Scientific Research Foundation for the Returned Overseas Chinese Scholars, State Education Ministry, China. 
pinning-controlled network synchronization and time-varying network synchronization, will be addressed. Emphasis will be on some basic theories, methodologies, conditions and criteria for the network synchronizability as well as some possible relationships between the network topology and the network synchronizability.

Keywords: Complex dynamical network, pinning control, synchronizability, synchronization, time-varying network topology.

\section{Introduction}

Complex networks are ubiquitous in the real world [4], [22], [26]. A complex network typically refers to an ensemble of dynamical units with nontrivial topological features that do not occur in simple systems such as completely regular lattices and completely random structures [4]. Typical examples of complex dynamical networks in point include the Internet, the World Wide Web, power grids, biological neural networks, trading market trains, scientific citation networks, social relationship networks, among many others. Over the past two decades, the theoretical study of complex dynamical networks has become a very active area of scientific research, inspired largely by the empirical studies on a huge number of real-world networks.

Collective behavior of complex dynamical networks, in particular, is a focal topic of considerable interest within science and technology communities, where one of the most important subjects is the network synchronization [1]-[39]. Synchronization is a timekeeping dynamical behavior requiring coordination of events to operate the entire network in unison. Synchronization is one of the basic motions in nature where many connected systems are evolving in synchrony. In fact, the uniform synchrony of coupled oscillators serves as a platform for the study, which can well explain many natural and technological phenomena [22].

Given some recent comprehensive surveys [1, 3, 4, 26, 31, 33, this Chapter aims at providing only a very brief account of some recent advances in the field of complex network synchronization, mainly from an engineering and technological perspective. Some commonly concerned issues in the study of network synchronization, especially pinning-controlled network synchronization and time-varying network synchronization, will be addressed. Emphasis will be on some basic theories, methodologies, conditions and criteria for the network synchronizability, as well as some possible relationships between the network topology and the network synchronizability, rather than empirical studies or application investigation, which have been reviewed in, e.g., [1, 3, 4].

The rest of the Chapter is organized as follows. Sections 2 introduces some basic concepts, theories and methodologies of complex network synchronization. Section 3 then addresses the pinning-controlled network synchronization 
problem. Section 4 further investigates the time-varying version of network synchronization. Some concluding remarks are finally given in Section 5.

\section{Problems of Network Synchronization}

Only linearly and diffusively coupled networks are considered in this Chapter for simplicity of presentation. Such a network consists of $N$ identical nodes, each being an $n$-dimensional autonomous dynamical system, described by [15]

$$
\dot{\mathbf{x}}_{i}=\mathbf{f}\left(\mathbf{x}_{i}\right)+\sum_{\substack{j=1 \\ j \neq i}}^{N} c_{i j}(t) \Gamma(t)\left(\mathbf{x}_{j}-\mathbf{x}_{i}\right), \quad i=1,2, \cdots, N
$$

where $t \geq t_{0}=0$ is the time variable, $\mathbf{f}: \mathbf{R}^{n} \rightarrow \mathbf{R}^{n}$ is continuously differentiable, $\mathbf{x}_{i}:=\mathbf{x}_{i}(t) \in \mathbf{R}^{n}$ is the state vector of node $i, \Gamma(t)$ is the inter-state coupling matrix between node $j$ and node $i(j \neq i ; 1 \leq i, j \leq N)$ at time $t, C(t)=\left[c_{i j}(t)\right]_{N \times N}$ is the configuration coupling matrix representing the topological structure of the network at time $t$, in which the coupling strengths $c_{i j}(t)$ are defined as follows: if there is a connection between node $i$ and node $j(j \neq i)$ at time $t$, then $c_{i j}(t) \neq 0$; otherwise, $c_{i j}(t)=0$; and the diagonal elements of $C(t)$ are defined by the diffusive coupling conditions:

$$
c_{i i}(t)=-\sum_{\substack{j=1 \\ j \neq i}}^{N} c_{i j}(t), \quad i=1,2, \cdots, N
$$

The above time-varying network can also be written in a compact form as 15

$$
\dot{\mathbf{x}}_{i}=\mathbf{f}\left(\mathbf{x}_{i}\right)+\sum_{j=1}^{N} c_{i j}(t) \Gamma(t) \mathbf{x}_{j}, \quad i=1,2, \cdots, N .
$$

As a special case, $\Gamma(t)$ can be a constant diagonal matrix of the form $\Gamma=$ $\operatorname{diag}\left\{\gamma_{1}, \gamma_{2}, \cdots, \gamma_{n}\right\}$, where $\gamma_{i} \geq 0$ are not all zero, $i=1,2, \cdots, N$, and $C(t)=c\left[a_{i j}\right]_{N \times N}$ for all time $t$, where $c$ is a constant coupling strength and $a_{i j}$ satisfy the following connectivity condition: if there is a connection between node $i$ and node $j(i \neq j)$, then $a_{i j}=a_{j i}=1$; otherwise, $a_{i j}=$ $a_{j i}=0$; and the diagonal elements are defined by the diffusive coupling conditions:

$$
a_{i i}=-\sum_{\substack{j=1 \\ j \neq i}}^{N} a_{i j}, \quad i=1,2, \cdots, N .
$$

In this case, the time-varying network (10) or (3) reduces to a simple timeinvariant model [25, 26, 27]: 


$$
\dot{\mathbf{x}}_{i}=\mathbf{f}\left(\mathbf{x}_{i}\right)+c \sum_{j=1}^{N} a_{i j} \Gamma \mathbf{x}_{j}, \quad i=1,2, \cdots, N
$$

Hereafter, assume that networks (3) and (5) are connected in the sense that there are no isolated clusters at all times. Thus, the coupling matrices $C(t)$ and $c\left[a_{i j}\right]$ are irreducible for all $t \geq 0$.

A mathematical definition of network synchronization is first given. Only complete synchronization is discussed here, again for simplicity of presentation.

Definition 1. 20] Network (3) is said to achieve synchronization if

$$
\lim _{t \rightarrow \infty}\left\|\mathbf{x}_{i}(t)-\mathbf{x}_{j}(t)\right\|=0 \quad \text { for all } i, j=1,2, \cdots, N,
$$

where $\|\cdot\|$ is the Euclidean norm.

In other words, by defining the synchronization manifold in the phase space as

$$
\left\{\mathbf{x}_{1}=\mathbf{x}_{2}=\cdots=\mathbf{x}_{N}\right\},
$$

the network synchronization is achieved if all state trajectories $\mathbf{x}_{i}(t)(1 \leq i \leq$ $N)$ converge into this manifold as $t \rightarrow \infty$.

Strictly based on this definition of network synchronization, some rigorous mathematical analysis on the network synchronizability and synchronization conditions can be carried out (see, for example, 17, 11, 12, 19, 35), which will be further reviewed below.

At this point, it is noted that Definition 1 is pure mathematical, namely, it does not concern about the ultimate synchronous behavior of the synchronized state vectors, wherever they arrive at. In practice, however, the purpose of studying network synchronization is to achieve something useful, for example to know where the network evolves to, when the synchronizing time is long enough, or better off, to ensure the network self-synchronize to a preferable and attainable target state. Of course, if this target state has no relations with the network, then this becomes a typical target-tracking problem in control theory. As further indicated in Remark 1 below, however, a self-synchronizing network will naturally evolve to one of its own solutions throughout the synchronization process continuously. Thus, from an engineering and technological application point of view, the above mathematical definition is very often being slightly twisted, as follows.

Definition 2. 2] Network (3) is said to achieve synchronization if

$$
\lim _{t \rightarrow \infty}\left\|\mathbf{x}_{i}(t)-\mathbf{s}(t)\right\|=0, \quad i=1,2, \cdots, N,
$$

for some $\mathbf{s}(t) \in \mathbf{R}^{n}$.

Remark 1. It may be possible that the network self-synchronize to a solution of its node system: $\dot{\mathbf{s}}(t)=\mathbf{f}(\mathbf{s}(t))$. For example, as a special case, when $\mathbf{s}(t)=\mathbf{s}$ 
is a constant vector, under some mild conditions to take a time limit on both sides of network (5) with the assumption that the network synchronization is indeed achieved, namely, if the network is able to self-synchronize to a constant vector: $\mathbf{x}_{i} \rightarrow \mathbf{s}(i=1,2, \cdots, N)$, then due to the diffusive condition (4), one has

$$
0=\dot{\mathbf{s}}=\mathbf{f}(\mathbf{s}) .
$$

This means that the constant state $\mathbf{s}$ is an equilibrium solution of the node equation of the network. Of course, if the network is unable to self-synchronize to a constant vector, then external control input is needed, a topic to be further discussed below.

In a more general situation, $\mathbf{s}(t)$ may even be a periodic or chaotic trajectory. But in the non-constant case the above time limiting process cannot be directly carried out; hence some rigorous working arguments are still needed to develop. As pointed out in [19], both variational analysis and linearization can be done only near the trajectory $\mathbf{s}(t)$ therefore this trajectory must contain an attracting set. Nevertheless, if external control input is allowed, then network controlled-synchronization to a pre-assigned target trajectory $\mathbf{s}(t) \in \mathbf{R}^{n}$ is often possible, as further discussed below.

Remark 2. In some previous publications, the above two definitions of network synchronization were not clearly distinguished, and often messed up. For instance, in [17, 18, the discussed network synchronization by nature belongs to the second type specified by Definition 2 above, but it was stated there as the first type described by Definition 1 instead.

\section{Basic Approaches and Results}

For simplicity of presentation, consider the complex dynamical network (5) and define $\mathbf{x}=\left[\mathbf{x}_{1}, \mathbf{x}_{2}, \cdots, \mathbf{x}_{N}\right], \mathbf{F}(\mathbf{x})=\left[\mathbf{f}\left(\mathbf{x}_{1}\right), \mathbf{f}\left(\mathbf{x}_{2}\right), \cdots, \mathbf{f}\left(\mathbf{x}_{N}\right)\right], \mathbf{A}=$ $\left[a_{i j}\right]_{N \times N}$, and $\bar{\Gamma}=\operatorname{diag}\{\Gamma, \Gamma, \cdots, \Gamma\}$. Then, the network can be rewritten as in the following matrix equation form:

$$
\dot{\mathbf{x}}=\mathbf{F}(\mathbf{x})+c \mathbf{A} \otimes \bar{\Gamma},
$$

where $\otimes$ is the Kronecker product.

To achieve network synchronization under Definition 1, a common approach is to apply the variational principle. Following 20, let $\xi_{i}$ be the variation of the state vector of the $i$ th node, $\mathbf{x}_{i}$, and introduce matrix $\xi=\left[\xi_{1}, \xi_{2}, \cdots, \xi_{N}\right]$. Then, one can obtain the following variational matrix equation:

$$
\dot{\xi}=\left[\mathbf{I}_{N} \otimes[D \mathbf{f}(\mathbf{x})]+c \mathbf{A} \otimes \bar{\Gamma}\right] \xi,
$$

where $[D \mathbf{f}(\mathbf{x})]$ is the Jacobian matrix at $\mathbf{x}$ and $\mathbf{I}_{N}$ is the $N$-dimensional identity matrix, in which each block is 


$$
\dot{\xi}_{k}=\left[[D \mathbf{f}(\mathbf{x})]+c \lambda_{k} \Gamma\right] \xi_{k},
$$

with $\lambda_{k}$ being the $k$ th eigenvalue of $\mathbf{A}, k=1,2, \cdots, N$. Obviously, the Jacobian matrix $[D \mathbf{f}(\mathbf{x})]$ here depends on the state vector $\mathbf{x}(t)$ for all $t \geq 0$, which is unknown beforehand and continuously evolves throughout the whole process.

To achieve network synchronization under Definition 2, a common approach is again to apply the variational principle, or directly linearize the network and then evaluate it at a state trajectory of the network. Following [2], one can obtain

$$
\dot{\xi}=\left[\mathbf{I}_{N} \otimes[D \mathbf{f}(\mathbf{s})]+c \mathbf{A} \otimes \bar{\Gamma}\right] \xi,
$$

where $[D \mathbf{f}(\mathbf{s})]$ is the Jacobian matrix evaluated at the trajectory $\mathbf{s}(t)$ which, as mentioned above, may be an equilibrium, periodic, or even chaotic trajectory. Similarly, each block of (13) is

$$
\dot{\xi}_{k}=\left[[D \mathbf{f}(\mathbf{s})]+c \lambda_{k} \Gamma\right] \xi_{k},
$$

with $\lambda_{k}$ being the $k$ th eigenvalue of $\mathbf{A}, k=1,2, \cdots, N$.

Let $\mathbf{A}_{L}=-\mathbf{A}$ be the Laplacian matrix of the network. Then, since the network is connected and diffusively coupled, 0 is an eigenvalue of $\mathbf{A}_{L}$ with multiplicity one and all the other eigenvalues of $\mathbf{A}_{L}$ are strictly positive, which are denoted by

$$
0=\lambda_{1}<\lambda_{2} \leq \lambda_{3} \leq \cdots \leq \lambda_{N} .
$$

Since the eigenvalue sets of $\mathbf{A}_{L}$ and $\mathbf{A}$ differ only by a minus sign, these two matrices will not be precisely distinguished below.

Now, consider the largest Lyapunov exponent $L_{\max }$ of network (5). This $L_{\max }$ is generally a function of the components and parameters of the network equation, such as $\mathbf{f}(\cdot), A, \Gamma$ and $c$. This $L_{\max }$ is usually referred to as the master stability function [20. Furthermore, given $\mathbf{f}(\cdot)$ and $\Gamma$, the region $S$ of negative real $\lambda_{k}$ where $L_{\max }$ is also negative is called the synchronized region 2. The synchronized solution of dynamical network (5) is locally asymptotically stable if

$$
-c \lambda_{k} \in S, \quad k=2,3, \cdots, N .
$$

For a given and fixed coupling strength $c>0$, if the synchronized region $S$ is unbounded, in the form of $(-\infty, \alpha]$ for some real constant $\alpha$, then the eigenvalue $\lambda_{2}$ of $\mathbf{A}$ characterizes the network synchronizability with respect to its topology. On the other hand, if the synchronized region $S$ is bounded, in the form of $\left[\alpha_{1}, \alpha_{2}\right]$ for some real constants $\alpha_{1} \leq \alpha_{2}$, then the eigenratio $r(A)=\lambda_{2} / \lambda_{N}$ of $\mathbf{A}$ characterizes the synchronizability. According to condition (16), the larger the $\left|\lambda_{2}\right|$ or the $r(A)$ is, the better the synchronizability will be, depending on the type of the synchronized region. 
The following discussions are based on Definition 1.

Consider network (5), where the coupling matrix $\mathbf{A}$ is symmetrical. Let

$$
\overline{\mathbf{x}}(t)=\frac{1}{N} \sum_{i=1}^{N} \mathbf{x}_{i}(t) .
$$

Then, the following result can be obtained.

Theorem 1. 19] Consider network (5). If all the following variational equations:

$$
\dot{\mathbf{w}}=\left[[D \mathbf{f}(\overline{\mathbf{x}}(t))]+\lambda_{k} \Gamma\right] \mathbf{w}, \quad k=2,3, \cdots, N,
$$

are exponentially stable, then the synchronization manifold (7) is locally exponentially stable, namely, the network synchronization is achieved locally and exponentially.

Actually, this result holds for networks in a slightly more general form, e.g., with a non-autonomous node function $\mathbf{f}(\cdot, t)$ [19]. Moreover, a corresponding global result can also be obtained [19].

Next, the following discussions are based on Definition 2.

As mentioned above, under Definition 2, in a general situation, $\mathbf{s}(t)$ may be (i) an equilibrium, (ii) a periodic trajectory, or (iii) a chaotic trajectory, as discussed respectively below.

(i) For an equilibrium $\mathbf{s}$

As mentioned above, under the assumption that the network synchronization is indeed achieved, namely, self-synchronizing to s constant vector, then this constant vector will be an equilibrium of the node system (see (9)). In this case, notice that in network model (18), the eigenvalues of the Jacobian matrix $[D \mathbf{f}(\overline{\mathbf{x}}(t))]$ are time functions, therefore the eigenvalue-region type of synchronization criterion (16) cannot be directly applied. For networks with an equilibrium $\mathbf{s}(t)=\mathbf{s} \in \mathbf{R}^{n}$, this synchronization criterion (16) turns out to be convenient to use, as shown by the following result.

Theorem 2. 10] In network (5), if the inter-state coupling matrix $\Gamma$ is antistable, namely, all its eigenvalues have positive real parts, then the network has an unbounded synchronized region about an equilibrium, in the form of $S=(-\infty,-\alpha)$ for some $\alpha>0$.

Note that the synchronized region $S$ may be bounded in the form of $\left(\alpha_{1}, \alpha_{2}\right) \subset$ $(-\infty, \infty)$ [2], or a union of some bounded and unbounded regions [6].

(ii) For a periodic trajectory $\mathbf{s}(t)$

For this case, the following concepts are useful.

Definition 3. 15] Let $\mathbf{s}(t)$ be a $T$-periodic solution $(T>0)$ of the node system $\dot{\mathbf{x}}=\mathbf{f}(\mathbf{x})$, with $\mathbf{x}(0)=\mathbf{x}_{0} \in \mathbf{R}^{n}$, and let $\gamma$ represent the closed orbit of $\mathbf{s}(t)$ in the state space: 


$$
\gamma=\{\mathbf{x} \mid \mathbf{x}=\mathbf{s}(t), \quad 0 \leq t<T\}
$$

If, for any $\varepsilon>0$, there exits a constant $\delta=\delta(\varepsilon)>0$ such that for any $\mathbf{x}_{0}$ satisfying

$$
d\left(\mathbf{x}_{0}, \gamma\right)=\inf _{\mathbf{x} \in \gamma}\left\|\mathbf{x}_{0}-\mathbf{x}\right\|<\delta
$$

a solution of the system, $\mathbf{s}(t)$, satisfies

$$
d(\mathbf{s}(t), \gamma)<\varepsilon, \quad \text { for all } t \geq 0,
$$

then this $T$-periodic solution trajectory, $\mathbf{s}(t)$, is said to be orbitally stable. Moreover, if there exist positive constants $\alpha, \beta$ and a real constant $h$ such that $\|\mathbf{x}(t-h)-\mathbf{s}(t)\| \leq \alpha e^{-\beta t}$ for $t \geq 0$, then $\mathbf{s}(t)$ is said to be orbitally asymptotically stable with an asymptotic phase $h$.

Definition 4. 15] Suppose that $\mathbf{s}(t)$ is a periodic solution of the node system $\dot{\mathbf{x}}=\mathbf{f}(\mathbf{x})$. Let $\gamma_{1}=1, \gamma_{2}, \cdots, \gamma_{n}$ be the Floquet multipliers of the variational equation of $\mathbf{s}(t), \dot{\mathbf{x}}=[D \mathbf{f}(\mathbf{s}(t))] \mathbf{x}$. Then, $\mathbf{s}(t)$ is said to be a hyperbolic periodic solution if $\left|\gamma_{j}\right| \neq 1$ for all $j=2, \cdots, n$. Moreover, $\mathbf{s}(t)$ is said to be a hyperbolic synchronous periodic solution of network (5) if all the Floquet multipliers of the variational equation of $\mathbf{s}(t)$ have absolute values less than 1 , except one multiplier which equals 1 .

Now, consider network (5) again.

Theorem 3. 15] Suppose that $\mathbf{s}(t)$ is a hyperbolic periodic solution of the node system $\dot{\mathbf{x}}=\mathbf{f}(\mathbf{x})$, and is orbitally asymptotically stable with a certain asymptotic phase. Suppose also that the network configuration coupling matrix $A=\left[a_{i j}\right]$ is diagonalizable. Then, $\mathbf{x}_{i}=\mathbf{s}(t)(i=1,2, \cdots, N)$ is a hyperbolic synchronous periodic solution of network (5) and is orbitally asymptotically stable with a certain asymptotic phase, if and only if all the following linear time-varying systems:

$$
\dot{\mathbf{w}}=\left[[D \mathbf{f}(\mathbf{s}(t))]+\lambda_{k} \Gamma\right] \mathbf{w}, \quad k=2, \cdots, N,
$$

are asymptotic stable about their zero solutions, where $\left\{\lambda_{k}\right\}$ are eigenvalues of the network coupling matrix $A$.

(iii) For a chaotic trajectory $\mathbf{s}(t)$

This case is difficult to analyze theoretically, due to the complexity of chaos. Nevertheless, under some "brute-force" types of conditions, such as the conservative LMI-type of conditions, some results can still be established, such as in 17. where however the approach of fixing $\mathbf{x}_{1}(t)=\mathbf{s}(t)$ is only heuristic but not completely rigorous.

There does not seem to be any rigorous result in the current literature on network synchronization to a chaotic trajectory, a solution of the chaotic node system of the network. A promising approach will be based on the profound 
chaos theory such as the ergodicity, an important and yet challenging topic for future research.

\section{Pinning-Controlled Network Synchronization}

Once again, consider network (5) for simplicity of presentation. The objective here is to let the network synchronize to some desired state, for example an equilibrium state $\mathbf{s} \in \mathbf{R}^{n}$.

If, under certain conditions as reviewed above, the network can selfsynchronize to $\mathbf{s} \in \mathbf{R}^{n}$, then no external control input is needed.

If, however, the network is unable to self-synchronize to $\mathbf{s} \in \mathbf{R}^{n}$, then one may apply some control input to the network to force it to achieve the objective. Since a complex network typically has a large number of nodes, so applying one controller to each node is clearly impractical or often impossible. A common practice is to apply pinning control onto only a small fraction $\delta$ $(0<\delta \ll 1)$ of the nodes in the network. Here, the name "pinning" means to let the controller stay there forever once it is applied at a node, in order to distinguish itself from others like self-organizing control and switching control.

Without loss of generality, suppose that the first $l$ nodes $(1,2, \cdots, l)$ are selected to pin, where $l=\lfloor\delta N\rfloor$ is the smaller but nearest integer to the real number $\delta N$. Then, referred to (5), the controlled network can be described by

$$
\begin{aligned}
& \dot{\mathbf{x}}_{k}=\mathbf{f}\left(\mathbf{x}_{k}\right)+c \sum_{j=1}^{N} a_{k j} \Gamma \mathbf{x}_{j}+\mathbf{u}_{k}, \quad k=1,2, \cdots, l, \quad(1 \leq l<N) \\
& \dot{\mathbf{x}}_{k}=\mathbf{f}\left(\mathbf{x}_{k}\right)+c \sum_{j=1}^{N} a_{k j} \Gamma \mathbf{x}_{j} \quad k=l+1, l+2, \cdots, N .
\end{aligned}
$$

For simplicity here, use linear state-feedback control:

$$
\mathbf{u}_{k}=-c d_{k} \Gamma\left(\mathbf{x}_{k}-\mathbf{s}\right), \quad k=1,2, \cdots, l,
$$

where $\left\{d_{k}\right\}$ are positive constant feedback gains to be determined. Let

$$
D=\operatorname{diag}\left\{d_{1}, d_{2}, \cdots, d_{l}, 0, \cdots, 0\right\}
$$

Theorem 4. 24 Suppose that there exists a constant $\rho>0$ such that $[[D \mathbf{f}(\mathbf{s})]+\rho \Gamma]$ is Hurwitz stable. Let $\lambda_{\max }$ be the largest eigenvalue of matrix $A-D$. If

$$
c \lambda_{\max } \leq \rho,
$$

then the equilibrium state $\mathbf{s}$ of the controlled network (5) is locally exponentially stable.

Theorem 5. 9 Assume that $\mathbf{f}(\mathbf{x})$ is Lipschitz continuous in $\mathbf{x}$ with Lipschitz constant $L_{c}^{f}>0$, and the node $\dot{\mathbf{x}}_{i}=\mathbf{f}\left(\mathbf{x}_{i}\right)$ is chaotic for all $i=1,2, \cdots, N$, 
with the maximum positive Lyapunov exponent $h_{\max }>0$ and $\lambda_{\min }$ being the minimum eigenvalue of the positive definite matrix $[-A+D]$ :

(i) if

$$
c>L_{c}^{f} / \lambda_{\min },
$$

then the controlled network (5), with $\Gamma=I$, is globally stable about the equilibrium s;

(ii) if

$$
c>h_{\max } / \lambda_{\min },
$$

then the controlled network (5) with $\Gamma=I$ is locally asymptotically stable about the equilibrium $\mathbf{s}$.

Remark 3. There were several closely-related papers on this approach to pinning control of network, e.g., [13.

For a more general form of networks, there are a couple of results reported in 9] where, however, Theorem 1 and its proof need some corrections: some involved matrices need to be symmetrical and the matrix $U$ therein needs to be a positive-definite diagonal matrix (as in [7]).

For some even more general cases, e.g., with time-varying topologies and (or) time-delayed couplings, see 28, 29, for which the network may even be pinned to a rather arbitrary trajectory (not limited to an equilibrium).

For networks with unknown topological parameters, adaptive pinning controllers may be employed, as further discussed below.

Consider a general complex dynamical network model of $N$ identical nodes, with linear and diffusive couplings, in the following form [36, 37]:

$$
\dot{\mathbf{x}}_{i}=\mathbf{f}\left(\mathbf{x}_{i}\right)+c \sum_{j=1}^{N} a_{i j} \Gamma \mathbf{x}_{j}+\mathbf{u}_{i}\left(\mathbf{x}_{1}, \cdots, \mathbf{x}_{N}\right),
$$

where the control inputs satisfy $\mathbf{u}_{i}(\mathbf{x}, \cdots, \mathbf{x})=0$. Let $\lambda_{i}^{m}$ be the maximum eigenvalues of matrix $A_{i}$, where $A_{i}$ is the $i$ th minor of the coupling matrix $A=\left[a_{i j}\right]$, obtained by removing the first $i-1$ rows and the first $i-1$ columns from $A, i=1,2, \cdots, N$.

Theorem 6. 36] Suppose that the Jacobian matrix of the network (25) is bounded, $\|[D \mathbf{f}(\mathbf{s})]\| \leq \alpha$ at a trajectory $\mathbf{s}(t) \in \mathbf{R}^{n}$ for all $t \geq 0$. If there is an integer $l, 1 \leq l<N$, such that $\lambda_{l+1}^{m}<-\alpha /\|\Gamma\|$, then the controlled network (25) is locally asymptotically stable about the trajectory $\mathbf{s}(t)$ under the following adaptive pinning controllers:

$$
\begin{cases}\mathbf{u}_{i}=-p_{i}\left(\mathbf{x}_{i}-\mathbf{s}\right), & \dot{p}_{i}=q_{i}\left\|\mathbf{x}_{i}-\mathbf{s}\right\|^{2}, \\ \mathbf{u}_{i}=0, & l \leq i \leq l, \\ & l+1 \leq i \leq N,\end{cases}
$$

where $q_{i}$ are some positive constants, $1 \leq i \leq l$. 
A more general setting of this problem and results can be found in 36 .

The simplest pinning control scheme is to use only one single controller (with $l=1$ ), namely, in (25) with $\mathbf{u}_{1}=c \varepsilon\left(\mathbf{x}_{1}(t)-\mathbf{s}(t)\right.$ ) and $\mathbf{u}_{i}(t)=0$ for all $i=2,3, \ldots, N$. Under some conditions (e.g., strong coupling strength $c$ ), this is possible, as shown in [7].

\section{Time-Varying Complex Network Models}

In this section, a network synchronization criterion for the time-varying network (3) is reviewed.

In network (3), assume that $\mathbf{f}: \Omega \rightarrow \mathbf{R}^{n}$ is continuously differentiable on $\Omega=\left\{\mathbf{x} \in \mathbf{R}^{n} \mid\|\mathbf{x}-\mathbf{s}(t)\|<r\right\}$. Suppose that the Jacobian matrix $[D \mathbf{f}(\mathbf{x})]$ is bounded and Lipschitz on $\Omega$ uniformly in $t$. Suppose also that there exists a real matrix, $\Phi(t)$, nonsingular for all $t \geq 0$, such that

$$
\Phi^{-1}(t) A(t) \Phi(t)=\operatorname{diag}\left\{\lambda_{1}(t), \lambda_{2}(t), \cdots, \lambda_{N}(t)\right\}
$$

and $\dot{\Phi}^{-1}(t) \Phi(t)=\operatorname{diag}\left\{\beta_{1}(t), \beta_{2}(t), \cdots, \beta_{N}(t)\right\}$.

Theorem 7. 15] Let $\mathbf{s}(t)$ be an exponentially stable solution of the node system $\dot{\mathbf{x}}=\mathbf{f}(\mathbf{x})$ in network (3). Then, all state vectors of the network will synchronize to $\mathbf{s}(t)$ if and only if all the following linear time-varying systems:

$$
\dot{\mathbf{w}}=\left[D \mathbf{f}(\mathbf{s}(t))+\lambda_{k}(t) \Gamma(t)-\beta_{k}(t) \mathbf{I}_{n}\right] \mathbf{w}, \quad k=2, \cdots, N,
$$

are exponentially stable about their zero solutions.

Remark 4. Theorem 7 indicates that synchronization of the time-varying dynamical network (3) is completely determined by its inter-state coupling matrix $\Gamma(t)$, and the eigenvalues (26) as well as the corresponding eigenvectors of the configuration coupling matrix $A(t)$, where $\beta_{k}(t)$ are functions of these eigenvectors. In comparison, the synchronization of the time-invariant dynamical network (5) is completely determined by its inter-state coupling matrix $\Gamma$ and the eigenvalues of the network-configuration coupling matrix $A[18,26]$.

\section{Conclusions}

This Chapter has presented a very brief overview of some progressive results on complex dynamical network synchronization from an engineering and technological perspective. Some commonly concerned issues in the current research on network synchronization, particularly on pinning-controlled network synchronization and time-varying network synchronization, have been addressed, with emphasis on some basic theories, methodologies, conditions 
and criteria for the network synchronizability as well as some possible relationships between the network topology and the network synchronizability.

In this Chapter, however, due to space limitation many other types of even more general networks have not been discussed, such as weighted, evolutionary, switching, delayed, clustering, and nonlinearly coupled networks, and many other interesting and important results have not been reviewed. Moreover, only complete synchronization has been discussed. Many other types of synchronization such as partial, cluster, projective, generalized, and phase synchronization have been left out. The interested readers may search for them in the following list of closely-related good references, therefore will not be further discussed here for brevity.

\section{Acknowledgement}

The authors thank Professor Tianping Chen for his valuable discussions and comments on some related technical issues.

\section{References}

1. Arenas, A., Diaz-Guilera, A., Kurths, J., Moreno, Y., Zhou, C.: Synchronization in complex networks. Phys. Rep. 469, 93-153 (2008)

2. Barahona, M., Pecora, L.M.: Synchronization in small-world systems. Phys. Rev. Lett. 89(5) art. 054101 (2002)

3. Boccaletti, S., Kurths, J., Osipov, G., Valladares, D.L., Zhou, C.S.: The synchronization of chaotic systems. Phys. Rep. 366, 1-101 (2002)

4. Boccaletti, S., Latora, V., Moreno, Y., Chavez, M., Hwang, D.-U.: Complex networks: Structure and dynamics. Phys. Rep. 424, 175-308 (2006)

5. Cai, S., Zhou, J., Xiang, L., Liu, Z.: Robust impulsive synchronization of complex delayed dynamical networks. Phys. Lett. A 372, 4990-4995 (2008)

6. Chen, G., Duan, Z.: Network synchronizability analysis: a graph-theoretic approach. Chaos 18, 037102 (2008)

7. Chen, T.P., Liu, X., Lu, W.: Pinning complex network by a single controller. IEEE Trans. Circ. Syst. I 54, 1317-1326 (2007)

8. Li, X.: Sync in complex dynamical networks: stability, evolution, control, and application. Int. J. Comput. Cognition 3, 16-26 (2005)

9. Li, X., Wang, X., Chen, G.: Pinning a complex dynamical network to its equilibrium. IEEE Trans. Circ. Syst.-I 51, 2074-2087 (2004)

10. Liu, C., Duan, Z., Chen, G., Huang, L.: Analyzing and controlling the network synchronization regions. Physica A 386, 531-542 (2007)

11. Liu, X., Chen, T.P.: Exponential synchronization of the linearly coupled dynamical networks with delays. Chin. Ann. Math., Ser. B 28, 737-746 (2007)

12. Liu, X., Chen, T.P.: Synchronization analysis for nonlinearly-coupled complex networks with an asymmetrical coupling matrix. Physica A 387, 4429-4439 (2008)

13. Lü, J.: Mathematical models and synchronization criterions of complex dynamical networks. Syst. Eng. Theory Pract. 24, 17-22 (2004) 
14. Lü, J.: Synchronization of complex networks: Theories, approaches, applications and outlook. Adv. Mech. 38, 713-722 (2008)

15. Lü, J., Chen, G.: A time-varying complex dynamical network model and its controlled synchronization criteria. IEEE Trans. Autom. Control. 50, 841-846 (2005)

16. Lü, J., Leung, H., Chen, G.: Complex dynamical networks: Modelling, synchronization and control. Dyn. Continuous Discret. Impul. Syst., Ser. B 11a, 70-77 (2004)

17. Lü, J., Yu, X., Chen, G.: Chaos synchronization of general complex dynamical networks. Physica A 334, 281-302 (2004)

18. Lü, J., Yu, X., Chen, G., Cheng, D.: Characterizing the synchronizability of small-world dynamical networks. IEEE Trans. Circ. Syst.-I 51, 787-796 (2004)

19. Lu, W., Chen, T.P.: New approach to synchronization analysis of linearly coupled ordinary diferential systems. Physica D 213, 214-230 (2007); A new approach to synchronization analysis of linearly coupled map lattices. Chin. Ann. Math. Ser. B 28, 149-160 (2007)

20. Pecora, L., Carroll, T.: Master stability functions for synchronized coupled systems. Phys. Rev. Lett. 80, 2109-2112 (1998)

21. Sorrentino, F., di Bernardo, M., Garofalo, F., Chen, G.: Controllability of complex networks via pinning. Phys. Rev. E 75, 046103 (2007)

22. Strogatz, S.H.: Sync: The Emerging Science of Spontaneous Order. Hyperion, New York (2003)

23. Strogatz, S.H., Stewart, I.: Coupled oscillators and biological synchronization. Sci. Amer. 12, 102-109 (1993)

24. Wang, X., Chen, G.: Pinning control of scale-free dynamical networks. Physica A 310, 521-531 (2002)

25. Wang, X., Chen, G.: Synchronization in scale-free dynamical networks: robustness and fragility. IEEE Trans. Circ. Syst.-I 49, 54-62 (2002)

26. Wang, X., Chen, G.: Complex networks: small-world, scale-free, and beyond. IEEE Circ. Syst. Mag. 3(1), 6-20 (2003)

27. Wang, X., Li, X., Chen, G.: Complex Networks Theory and Its Applications. Tsinghua Univ. Press, Beijing (2006)

28. Xiang, L., Chen, Z.Q., Liu, Z., Chen, F., Yuan, Z.: Pinning control of complex dynamical networks with heterogeneous delays. Computers Math. Appl. 56, 1423-1433 (2008)

29. Xiang, L., Liu, Z., Chen, Z.Q., Yuan, Z.: Pinning weighted complex networks with heterogeneous delays by a small number of feedback controllers. Sci. China, Ser. F 51, 511-523 (2008)

30. Yu, W., Cao, J., Lü, J.: Global synchronization of linearly hybrid coupled networks with time-varying delay. SIAM J. Appl. Dyn. Syst. 7, 108-133 (2008)

31. Wu, C.W.: Synchronization in Coupled Chaotic Circuits and Systems. World Scientific, Singapore (2002)

32. Wu, C.W.: Synchronization and convergence of linear dynamics in random directed networks. IEEE Trans. Autom. Control 51, 1207-1210 (2006)

33. Wu, C.W.: Synchronization in Complex Networks of Nonlinear Dynamical Systems. World Scientific, Singapore (2007)

34. Zhang, Q., Lu, J.A., Lü, J., Tse, C.K.: Adaptive feedback synchronization of a general complex dynamical network with delayed nodes. IEEE Trans. Circ. Syst.-II 55, 183-187 (2008) 
35. Zhou, J., Chen, T.P.: Synchronization in general complex delayed dynamical networks. IEEE Trans. Circ. Syst.-I 53, 733-744 (2006)

36. Zhou, J., Lu, J.A., Lü, J.: Adaptive synchronization of an uncertain complex dynamical network. IEEE Trans. Autom. Control 51, 652-656 (2006)

37. Zhou, J., Lu, J.A., Lü, J.: Pinning adaptive synchronization of a general complex dynamical network. Automatica 44, 996-1003 (2008)

38. Zhou, J., Lu, J.A., Lü, J.: Erratum to: Pinning adaptive synchronization of a general complex dynamical network. Automatica 45, 598-599 (2009); Automatica 44, 996-1003 (2008)

39. Zhou, J., Xiang, L., Liu, Z.: Synchronization in complex delayed dynamical networks with impulsive effects. Physica A 384, 684-692 (2007) 